\title{
A Study on the Void Formation in Residual Wall Thickness of Fluid-Assisted Injection Molding Parts
}

\author{
Hyung-Pil Park, ${ }^{1}$ Baeg-Soon Cha, ${ }^{1}$ Soo-Bin Park, ${ }^{2}$ Jae-Hyuk Choi, ${ }^{2}$ Dong-Han Kim, \\ Byung-Ohk Rhee, ${ }^{2}$ and Kye-Hwan Lee ${ }^{3}$ \\ ${ }^{1}$ Molding \& Forming Technology R\&D Group, Korea Institute of Industrial Technology, 7-46 Songdo-dong, Yeonsu-gu, \\ Incheon 406-840, Republic of Korea \\ ${ }^{2}$ Department of Mechanical Engineering, Ajou University, San 5 Woncheon-dong, Yeongtong-gu, Suwon, \\ Kyungki-do 443-749, Republic of Korea \\ ${ }^{3}$ Department of Manufacturing Engineering, University of Texas Pan-American, Edinburg, TX 78539, USA
}

Correspondence should be addressed to Hyung-Pil Park; php76@kitech.re.kr

Received 23 December 2013; Accepted 22 April 2014; Published 9 June 2014

Academic Editor: Mohd Sapuan Salit

Copyright (C) 2014 Hyung-Pil Park et al. This is an open access article distributed under the Creative Commons Attribution License, which permits unrestricted use, distribution, and reproduction in any medium, provided the original work is properly cited.

In fluid-assisted injection molding, the distribution of the residual wall thickness on the inside and outside of the curved area is different, and void is formed due to the effect of the shrinkage on the outside where the residual wall thickness is thicker. The shrinkage that takes place in the residual wall is affected by the rheological changes in the polymer caused by temperature change and also by the thermal properties of the penetration fluid. In this study, the different effects on void formation in residual wall during fluid-assisted injection molding were analyzed, and water and silicone oil that had different thermal properties were used for the fluids. For this, heat transfer analysis and injection molding analysis were conducted. The void formation occurred due to the different temperature distribution and volumetric shrinkage in the direction of the residual wall in the curved area with a hollow section. It was also found that the void formation in the curved area decreased in the case of silicone oil compared to the case of water from simulation and experiments.

\section{Introduction}

Fluid-assisted injection molding is a process where the melt polymer is filled into a cavity after which the fluid penetrates to form a hollow space inside the molded product [1-3]. Currently, the commercialized fluid-assisted injection molding processes are the gas-assisted injection molding in which gas is used as the penetration fluid and water-assisted injection molding in which water is used as the penetration fluid. The water-assisted injection molding technique is applied in manufacturing three-dimensional tubular parts with a hollow cross-section that has recently been provided with functionality. Compared to gas, water has the advantage of being able to reduce the cooling cycle by at least $30 \%$, since it has 4 times higher specific heat and more than 40 times higher thermal conductivity. In particular, it is a technique that is appropriate for manufacturing hollow products with functionality because of its advantages of forming uniformed thickness in a hollow area, smooth internal part surface, larger spaces, and longer hollow sections compared to gasassisted injection molding [4]. However, despite numerous benefits in water-assisted injection molding, it has the problem of having a void present in the residual wall where the hollow section forms due to the effects of the thermal conductivity of water $[5,6]$.

Void that forms inside during the formation of the hollow section is mainly due to two causes. First is the void that occurs due to the unstable flow of the fluid in forming the hollow section and second is the void due to the shrinkage that is present because of the difference in residual wall thickness between the inside and the outside portion of the product, which forms in the curved area during the formation of the hollow section.

According to a study by Liu et al. $[7,8]$ on the occurrence of void in water-assisted injection molding, the void which forms in the residual wall of the hollow section is affected 


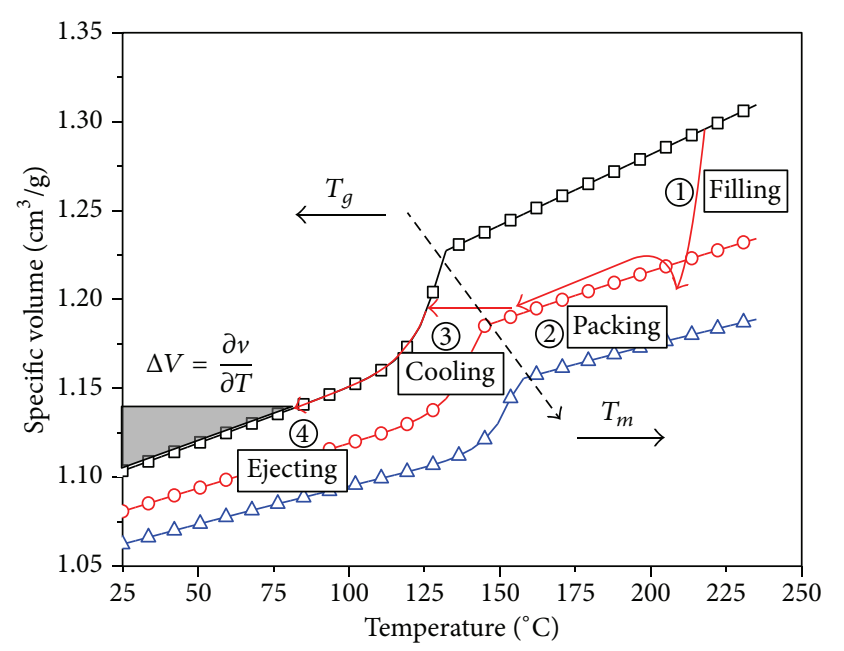

$$
\begin{aligned}
& \multimap-P=0(\mathrm{MPa}) \\
& \multimap-P=50(\mathrm{MPa}) \\
& \neg-P=100(\mathrm{MPa})
\end{aligned}
$$

FIgUre 1: Trace of an injection molding cycle in a PvT diagram.

by the semicrystalline polymer in which there is a large volumetric shrinkage present and also by the pressure and velocity of water injection. Furthermore, the difference in shrinkage increases even more in case the glass fiber is added for reinforcement of strength, causing larger formations of void in the residual wall [9].

In general, approximately $20 \%$ of specific volume changes during the process due to changes in the temperature and pressure of the polymer. Such change in specific volume generates shrinkage in the molding part, and the volumetric shrinkage that mostly occurs during the cooling process affects the dimensional accuracy and quality of the molding product.

Figure 1 shows the PvT data of the polypropylene, which is a typical semicrystalline plastic. Once polymer filling takes place, it shifts to position (1) because of the change in specific volume due to the increased injection pressure. Once packing pressure is in action after completion of filling, the temperature and pressure decrease due to the effects of the solidification of the polymer and the specific volume changes according to the curve of (2). After the completion of the packing pressure, the pressure of the molding product drops to atmospheric pressure and undergoes stages (3) and (4) at which great change in specific volume takes place as it passes through the melting temperature and glass transition temperature zones. The volumetric shrinkage in the final molding product is determined by the difference between the specific volume during product ejection and the specific volume at free air temperature. Therefore, greater shrinkage takes place as the ejecting temperature increases [10].

Figure 2 shows the conceptual diagram that illustrates the mechanism of void formation occurring from the effects of shrinkage inside the molding product. In a study by Aida and Hazawa [11], it was experimentally proven that the void, which forms due to shrinkage, first grows rapidly due to internal shrinkage but there is no void growth after a

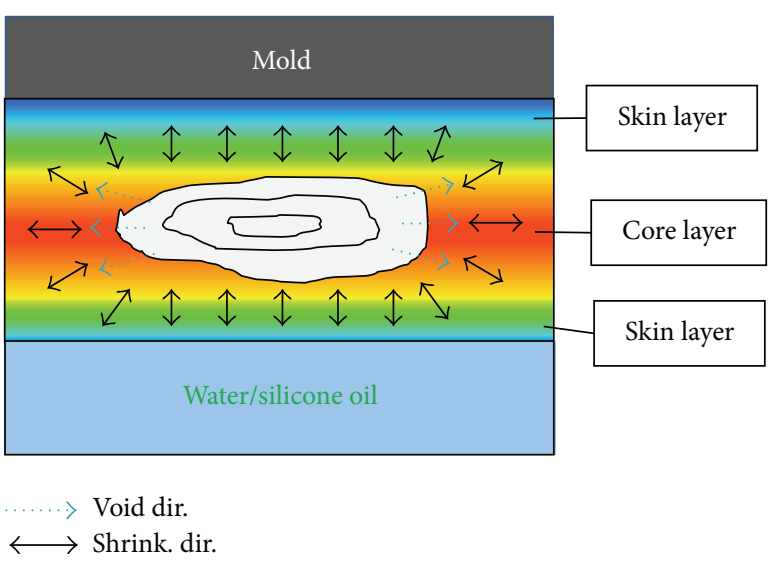

FIGURE 2: Mechanism of void formation.

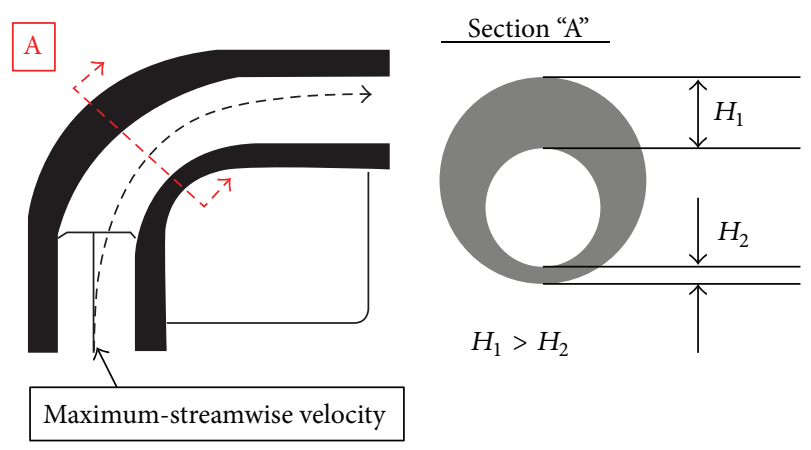

FIgURE 3: The effect of maximum streamwise velocity.

certain period of cooling time. There has also been research conducted to predict the growth of void due to shrinkage. Kim [12] and Ahn and Kim [13] proposed a theoretical model on the length of void growth by shrinkage, proving that void growth can be predicted by comparing the theoretical model with experimental results.

In this study, the effects of using water and silicone oil that have different thermal properties from one another on the void formation in the residual wall thickness during the fluidassisted injection molding process were analyzed. To do this, void formation that occurred due to the effects of shrinkage was analyzed after analyzing the temperature and volumetric shrinkage distribution in the direction of the residual wall thickness of the hollow section by conducting heat transfer analysis and injection molding analysis. In addition, the effects of water and silicone oil on the void formation in the residual wall of the curved area were compared through fluidassisted injection molding experiments.

\section{Fluid-Assisted Injection Molding Process}

In fluid-assisted injection molding, a certain quantity of polymer is injected into the mold through injection molding process followed by a certain period of standby time to form a polymer frozen layer, after which fluid is injected to form the hollow section. This process is followed by another process for 


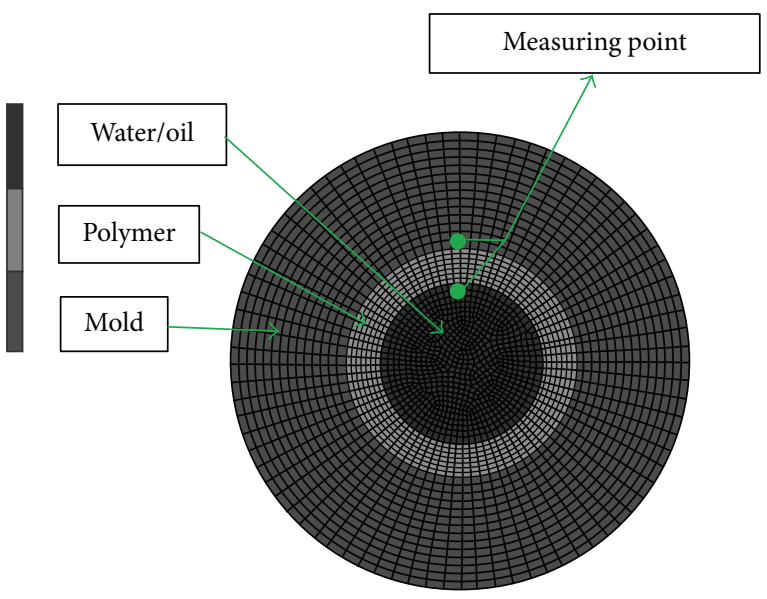

FIgURE 4: Definition of the heat transfer analysis model.

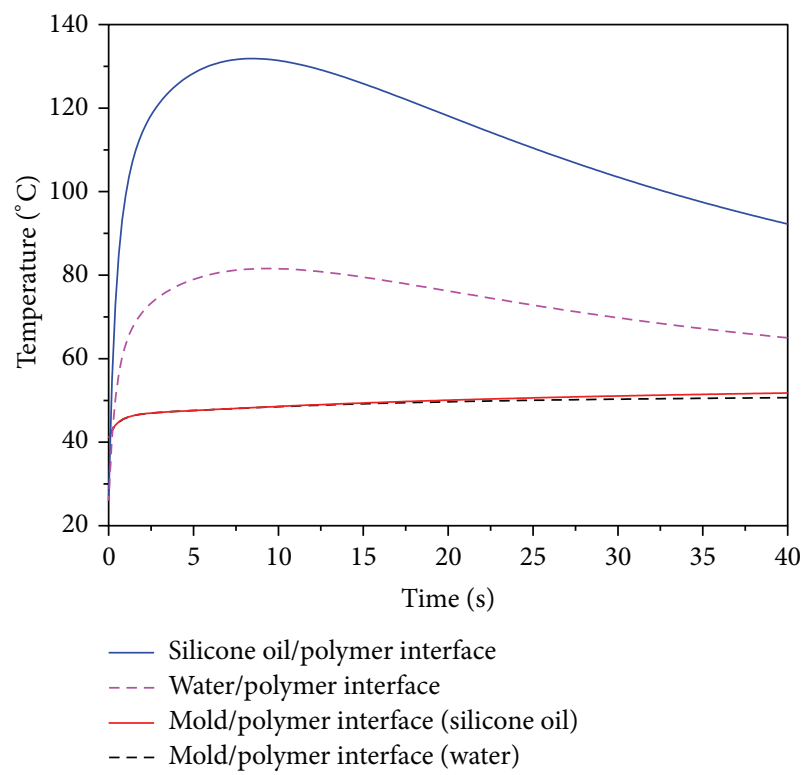

FIGURE 5: Result of heat transfer analysis for water and silicone oil.

the removal of fluid that remains inside the molding product, after which the final product is ejected.

However, during the injection of the fluid for the formation of the hollow section, there is uniformed distribution of residual wall thickness in the straight area but there is thickness deviation present in the curved area. Such effect is due to the fluid flowing according to the maximum streamwise velocity in Figure 3, with the outer thickness becoming thicker than the inner thickness because of the effects of centrifugal force generated by the fluid flowing through the curved area.

Once the fluid is injected, the polymer frozen layer is formed in the inner wall of the hollow section and this frozen layer rapidly grows through the effects of the thermal properties of the fluid. Because the formed frozen layer has high mechanical stiffness, the injection pressure of the fluid for compensating the shrinkage in the thickness direction of
TABLE 1: Thermal property for mold, water, and silicone oil.

\begin{tabular}{lccc}
\hline & $\begin{array}{c}\text { Density } \\
{\left[\mathrm{g} / \mathrm{cm}^{3}\right]}\end{array}$ & $\begin{array}{c}\text { Specific heat } \\
{\left[\mathrm{J} / \mathrm{kg} \cdot{ }^{\circ} \mathrm{C}\right]}\end{array}$ & $\begin{array}{c}\text { Thermal conductivity } \\
{\left[\mathrm{W} / \mathrm{m} \cdot{ }^{\circ} \mathrm{C}\right]}\end{array}$ \\
\hline Mold & 0.780 & 460 & 29 \\
Water & 0.988 & 4180 & 0.643 \\
Silicone oil & 0.9676 & 1500 & 0.16 \\
\hline
\end{tabular}

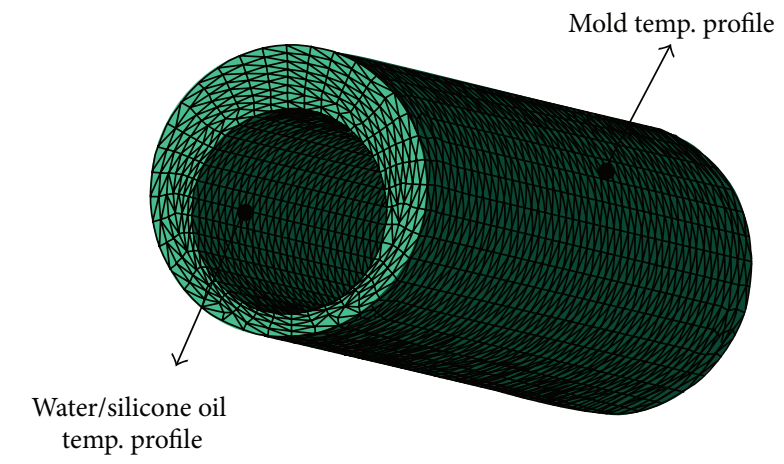

FIGURE 6: Finite element model for prediction of void generation.

the residual wall is not effective. Consequently, void forms in the curved area where the residual wall thickness is thick due to the effects of shrinkage.

\section{CAE Analysis for the Prediction of Void Formation}

In order to improve the void formation in the residual wall thickness during the formation of the hollow section through fluid-assisted injection molding, there must be a modification of the cooling properties using the thermal properties of the penetration fluid instead of optimizing the process parameters of the penetration fluid. Accordingly in this study, the effects of applying water and silicone oil, which have different thermal properties from one another, on the void formation inside the residual wall thickness were analyzed. To do this, the temperature of the penetration fluid was predicted through the heat transfer analysis, which was applied as the boundary condition for the injection molding analysis to analyze void formation due to shrinkage that is present in the residual wall thickness.

3.1. Heat Transfer Analysis. Heat transfer analysis was conducted by using commercial software MSC Marc 2011 in order to predict the temperature distribution between the hollow section and the penetration fluid during the fluid-assisted injection molding using water and silicone oil. Figure 4 shows that the finite element analysis model that was applied in the analysis and the thermal properties of the fluids defined in Table 1 were applied in the analysis as well. In this analysis, the boiling effect of water was not considered because it was assumed that the void formed during the cooling process after the completion of fluid injection. 

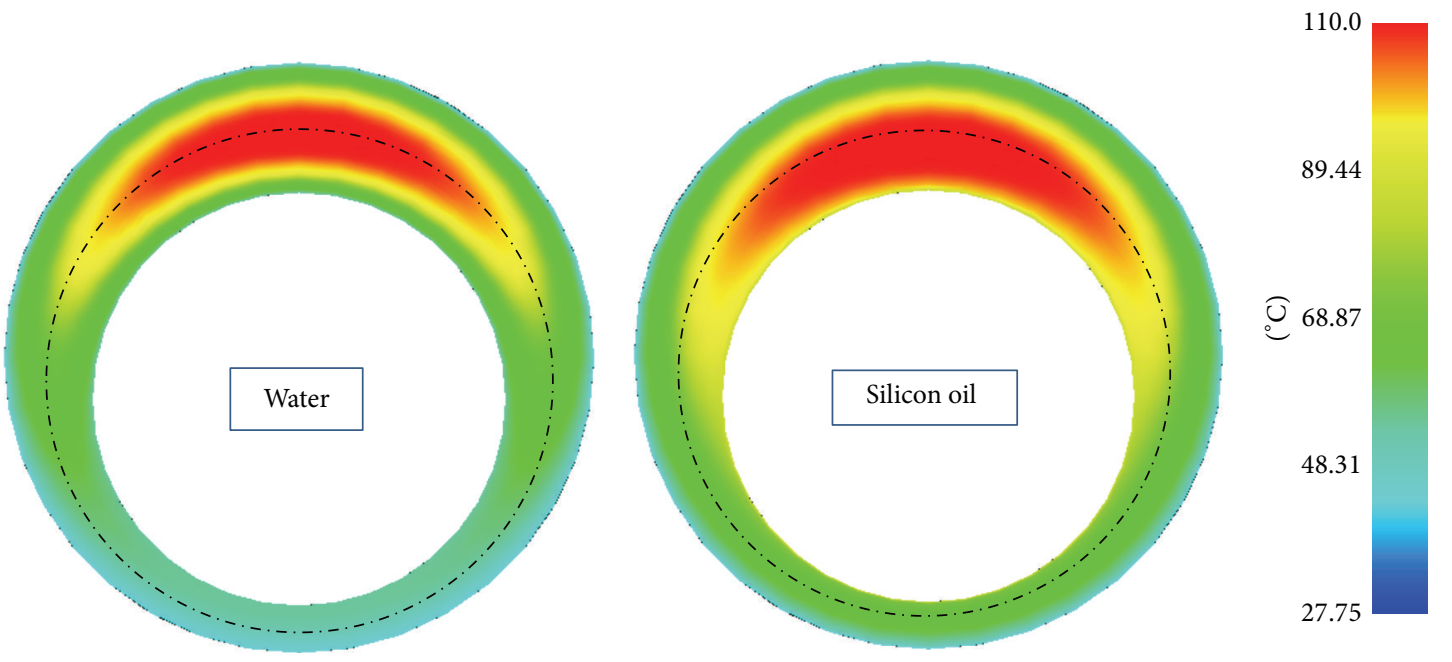

FIGURE 7: Distribution of temperature at the hollow part section.

In general, heat transfer coefficient can be calculated in many different ways but the heat transfer coefficient applied in this analysis is defined as follows [14]:

$$
\begin{aligned}
\mathrm{Nu} & =\frac{h D}{K_{f}}=0.664 \sqrt{\operatorname{Re}_{d}} \operatorname{Pr}^{1 / 3}, \\
\mathrm{Re}_{d} & =\frac{\rho U_{\infty} D}{u}, \\
h & =\frac{0.664 K_{f} \sqrt{\rho U_{\infty} D / u} \operatorname{Pr}^{1 / 3}}{D} .
\end{aligned}
$$

The heat transfer coefficient value is affected by the thermal conductivity coefficient, viscosity coefficient, fluid velocity, density, and more, among which the velocity of the fluid is one of the most sensitively reacting factors for the heat transfer coefficient. The results measured by using the linear potentiometer of the fluid-assisted injection molding system through experiment were applied for the velocity of the fluid. Velocities of $0.264 \mathrm{~m} / \mathrm{s}$ and $0.085 \mathrm{~m} / \mathrm{s}$ for water and silicone oil, respectively, were applied as the average velocity of the fluid that flowed while forming the hollow section. By calculating the heat transfer coefficient of water and silicone oil through (1), $4100 \mathrm{~W} / \mathrm{m}^{2 \circ} \mathrm{C}$ and $550 \mathrm{~W} / \mathrm{m}^{2 \circ} \mathrm{C}$ were applied in the analysis for water and silicone oil, respectively.

Figure 5 shows the results of the heat transfer analysis for water, silicone oil, and mold. It can be observed that higher temperature is reached in case of silicone oil compared to water due to the effects of different thermal properties.

3.2. Injection Molding Analysis with the Application of Heat Transfer Analysis Results. The injection molding analysis was conducted by applying the results of the heat transfer analysis of the penetration fluid and the mold temperature. Because this analysis was aimed at analyzing the void formation that takes place during the cooling process after the formation of the hollow section by the penetration fluid, it was defined to have thickness deviation present as shown in Figure 6. $\mathrm{M} 540(\mathrm{PP})\left(\mathrm{T}: 230^{\circ} \mathrm{C}, T_{g}: 116^{\circ} \mathrm{C}\right)$ of GS Caltex was applied as

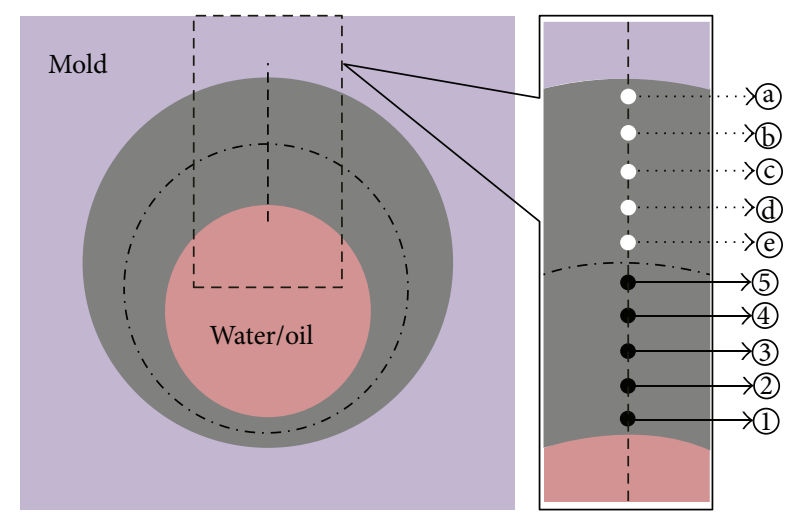

FIGURE 8: Set of measurement position for thickness direction.

a polymer for the analysis. The same process conditions were applied for both water and silicone oil and the results of the heat transfer analysis were used for the temperatures of the inner surface where the hollow section was formed and the outer surface that was in contact with the mold.

Figure 7 shows the temperature distribution in the thickness direction of the residual wall when applying the results of heat transfer analysis of water and silicone oil as the surface temperature boundary condition of the injection molding process. While both cases of using water and silicone oil show similar temperature distribution until the center of the residual wall thickness for the outer surface of the hollow section that is in contact with the mold, it can be observed for the inner surface of the hollow section that is in contact with water or silicone oil that the silicone oil has more uniformed temperature distribution until the center of the residual wall thickness compared to that of water. Such difference in temperature distribution signifies that there is difference in volumetric shrinkage present.

In order to analyze the change in volumetric shrinkage that takes place in the thickness direction of the residual wall in the hollow section, measuring locations for the outer 


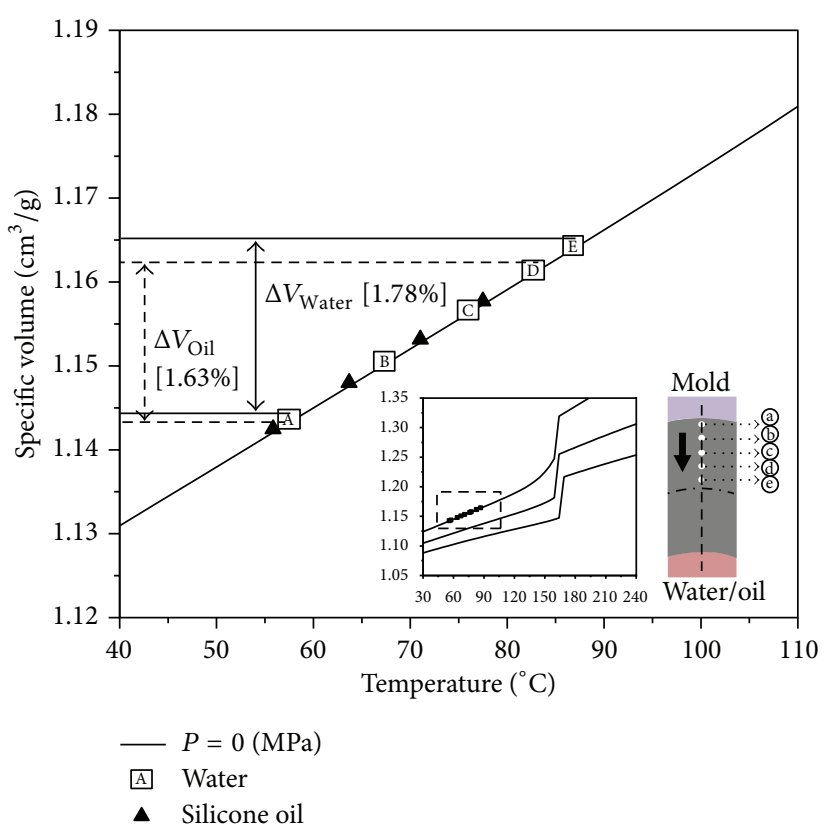

(a) From center of thickness to outer surface

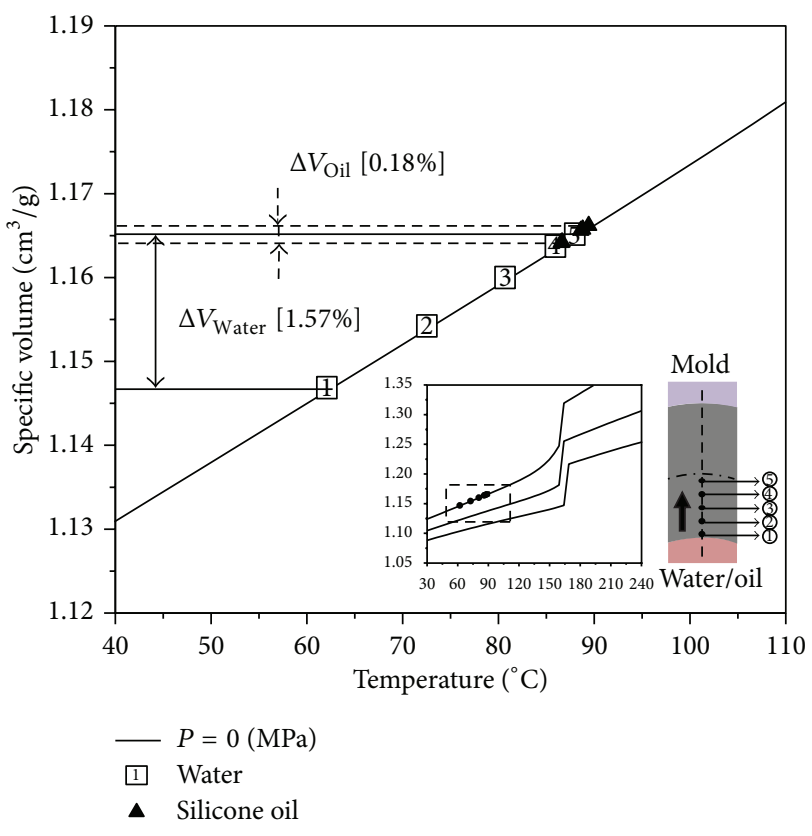

(b) From center of thickness to inner surface

FIGURE 9: Temperature versus specific volume for hollow part thickness.

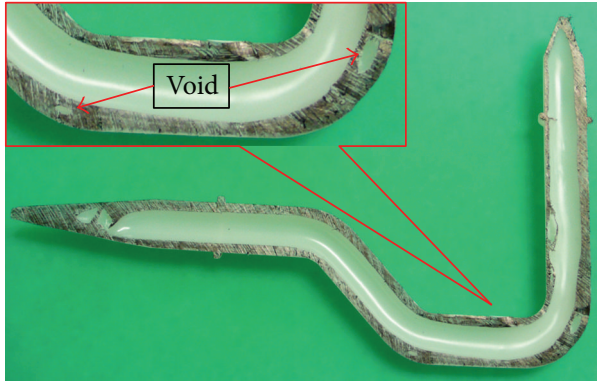

(a) Water

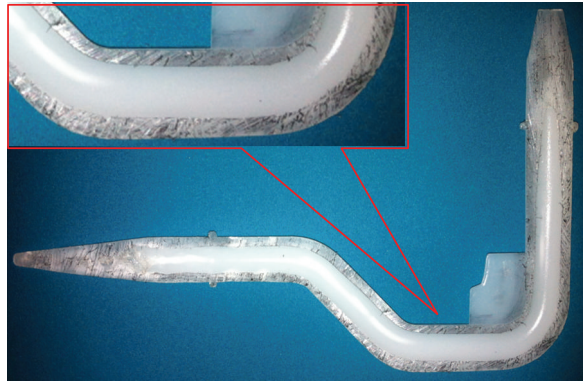

(b) Silicone oil

FIGURE 10: Comparison of experiment result between water and silicone oil.

and inner surfaces by having the center of the residual wall thickness as the basis were defined as shown in Figure 8.

Figures 9(a) and 9(b) show the changes in temperature and volumetric shrinkage according to the positions in the thickness direction of the residual wall. Figure 9(a) shows the change of volumetric shrinkage in the thickness direction from the center of the residual wall thickness to the area in contact with the mold. It is evident that the difference of volumetric shrinkage is not big for both cases since the results were $1.78 \%$ and $1.63 \%$ for water and silicone oil, respectively. Figure 9(b) shows the change of volumetric shrinkage in the thickness direction from the center of the residual wall thickness to the area in contact with the penetration fluid. It can be observed that there is significant difference in volumetric shrinkage since the results were $1.57 \%$ for water and $0.18 \%$ for silicone oil. This signifies that the thermal properties of water and silicone oil greatly affect the change in volumetric shrinkage.
Accordingly, it was possible to predict that the void was generated in the inner wall in case of water because there was significant difference in volumetric shrinkage between the center of the residual wall thickness and the inner/outer areas. On the other hand, it was possible to predict that such effects on void generation in case of oil were relatively lower since oil had less volumetric shrinkage difference compared to water.

\section{Fluid-Assisted Injection Molding Experiment}

Figure 10 shows the experiment results of the fluid-assisted injection molding using water and silicone oil. In case of water, void was generated on the outer surface of the curved area that formed the thick residual wall while the void did not form in case of silicone oil unlike water. This is understood 
to be the result of the volumetric shrinkage difference of the residual wall in the hollow section that is present due to the effects of the thermal properties of the fluid as proposed by the analysis.

\section{Conclusions}

It was understood that the difference in the outer and inner residual wall thickness in a curved area appeared differently according to the type of penetration fluid during the formation of the hollow section in fluid-assisted injection molding and that the void is generated on the outer surface where the residual wall thickness formed thicker. Through experimentation and analysis, it was evident that the effects of high specific heat and thermal conductivity of water resulted in the fast formation of the frozen layer in the inner wall that hindered the compensation of the volumetric shrinkage by the fluid penetration pressure, because of which void was generated due to the large volumetric shrinkage difference in the thickness direction. On the other hand, decreased void generation was confirmed in case of the silicone oil that had low specific heat and thermal conductivity since the formation of frozen layer in the inner wall was delayed, which resulted in smooth volumetric shrinkage compensation by fluid penetration pressure and decreased volumetric shrinkage difference in the thickness direction. It was understood through this study that the void formation in the residual wall thickness was affected by the thermal properties of the penetration fluid in the fluid-assisted injection molding process.

\section{Conflict of Interests}

The authors declare no competing financial interest.

\section{Acknowledgment}

This research was financially supported by development of manufacturing site greening technology project of Small and Medium Business Administration (SMBA).

\section{References}

[1] M. Knights, "Water injection molding makes hollow parts faster, lighter," Plastics Technology, vol. 48, no. 4, pp. 42-47, 2002.

[2] H. P. Heim and H. Potente, Specialized Molding Techniques, Plastics Design Library, New York, NY, USA, 2001.

[3] W. Michaeli, T. Jüntgen, and A. Brunswick, "WIT-en route to series production: first industrial application of the water injection technique," Kunststoffe Plast Europe, vol. 91, no. 3, pp. 37-39, 2001.

[4] S.-J. Liu and Y.-S. Chen, "Water-assisted injection molding of thermoplastic materials: effects of processing parameters," Polymer Engineering and Science, vol. 43, no. 11, pp. 1806-1817, 2003.

[5] S. Y. Yang and H. L. Chou, "Study on the residual wall thickness at dimensional transitions and curved sections in gas-assisted molded circular tubes," Polymer Engineering and Science, vol. 42, no. 1, pp. 111-119, 2002.
[6] K.-Y. Lin, F.-A. Chang, and S.-J. Liu, "Using differential mold temperatures to improve the residual wall thickness uniformity around curved sections of fluid assisted injection molded tubes," International Communications in Heat and Mass Transfer, vol. 36, no. 5, pp. 491-497, 2009.

[7] S.-J. Liu and C.-H. Lin, "An experimental study of water-assisted injection molding of plastic tubes with dimensional transitions," Journal of Reinforced Plastics and Composites, vol. 26, no. 14, pp. 1441-1454, 2007.

[8] S.-J. Liu and W.-K. Chen, "Experimental investigation and numerical simulation of cooling process in water assisted injection moulded parts," Plastics, Rubber and Composites, vol. 33, no. 6, pp. 260-266, 2004.

[9] S.-J. Liu, M.-J. Lin, and Y.-C. Wu, "An experimental study of the water-assisted injection molding of glass fiber filled polybutylene-terephthalate (PBT) composites," Composites Science and Technology, vol. 67, no. 7-8, pp. 1415-1424, 2007.

[10] G. Menges, W. Michaeli, and P. Mohren, How to Make Injection Molds, Hanser, Cincinnati, Ohio, USA, 2000.

[11] H. Aida and R. Hazawa, "Injection molding method for manufacturing a thermoplastic part free from sink marks using a void inducing member," US patent no. 5232654, August 1993.

[12] D. H. Kim, "Partial frame process technology," Polymer Science and Technology, vol. 7, no. 3, pp. 303-309, 1996.

[13] K. H. Ahn and D.-H. Kim, "Mechanism of void growth in the partial frame process," Polymer Engineering and Science, vol. 38, no. 10, pp. 1708-1715, 1998.

[14] F. P. Incropera and D. P. DeWitt, Fundamentals of Heat and Mass Transfer, John Wiley \& Sons, New York, NY, USA, 2006. 

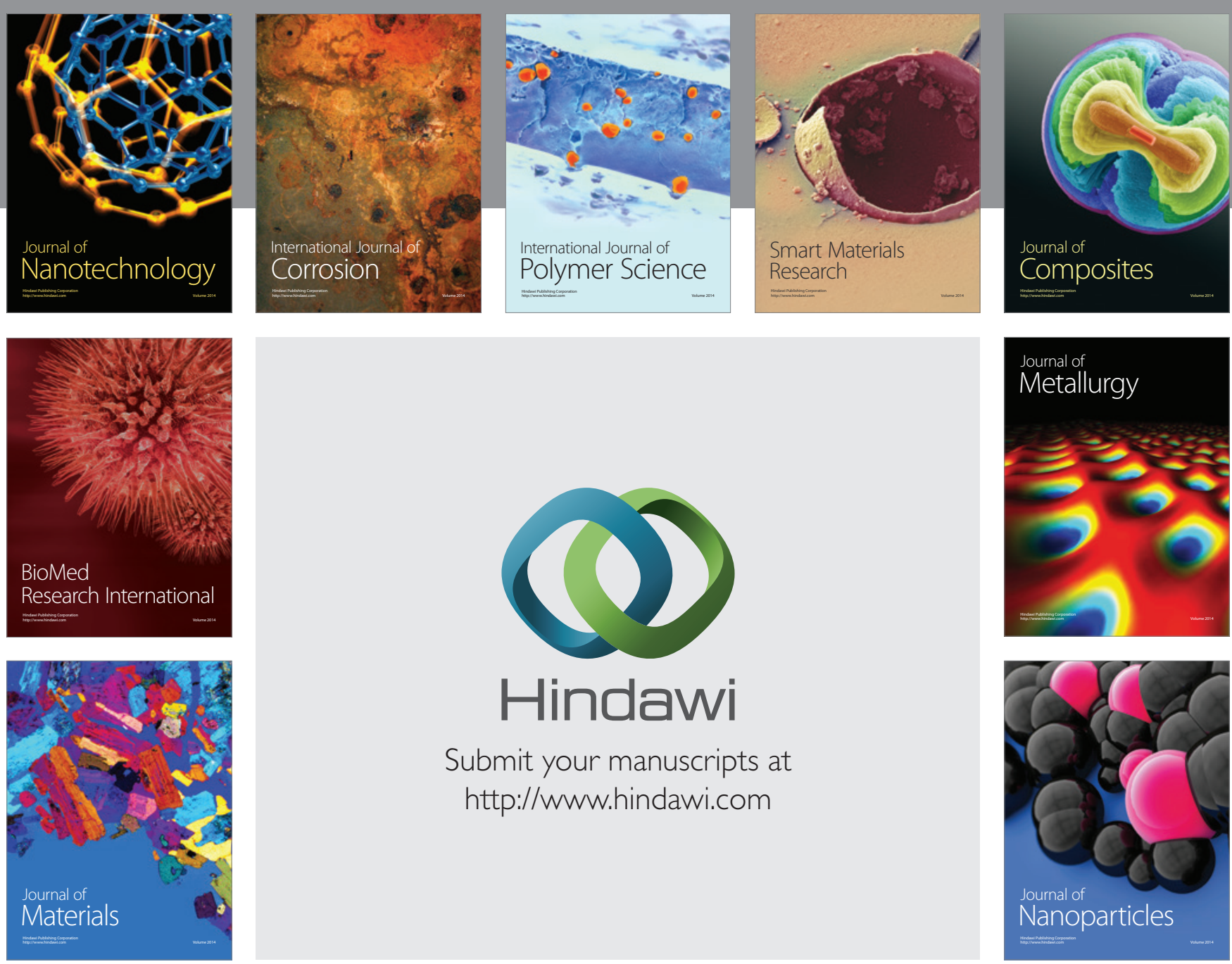

Submit your manuscripts at http://www.hindawi.com
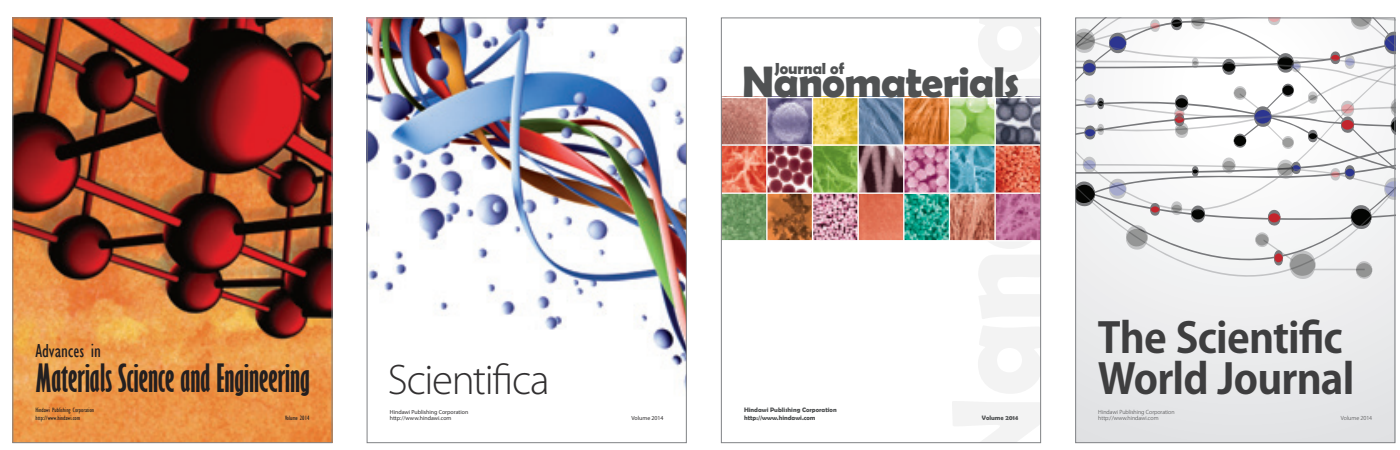

\section{The Scientific World Journal}
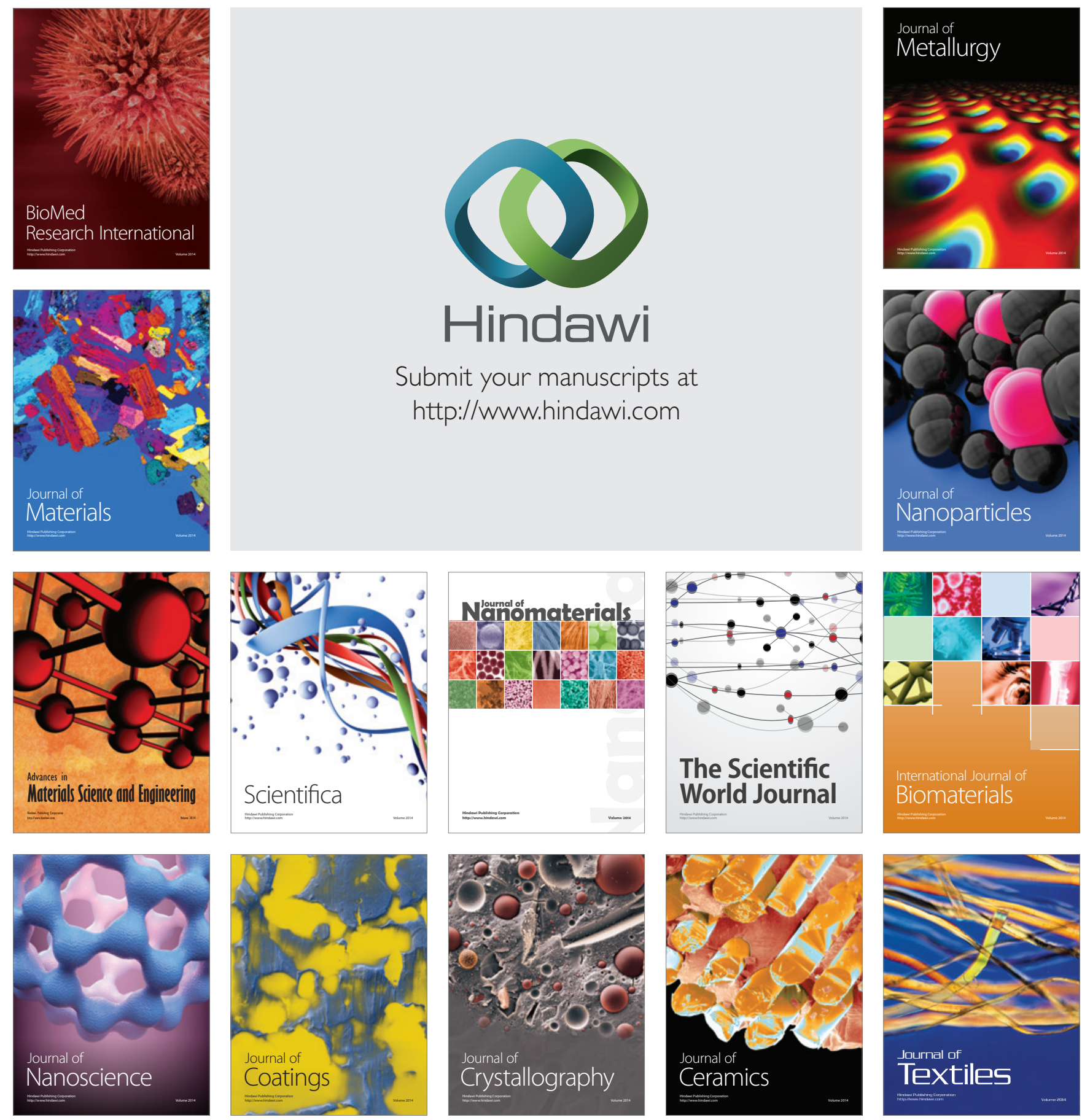